\title{
Erratum: Nonlinear causality of general first-order relativistic viscous hydrodynamics [Phys. Rev. D 100, 104020 (2019)]
}

Fábio S. Bemfica, Marcelo M. Disconzi, and Jorge Noronha

(Q) (Received 22 February 2022; published 3 March 2022)

DOI: $10.1103 /$ PhysRevD.105.069902

An additional condition for causality was missing in this paper. The condition below must be added in Eqs. (7), namely,

$$
2 \lambda \chi_{1} \geq c_{s}^{2} \lambda \chi_{2}+\lambda \chi_{3}+\chi_{2} \chi_{3}-\chi_{1}\left(\chi_{4}-\frac{4}{3} \eta\right)
$$

Furthermore, a correction is needed in the special case when $c_{s}=0$. The correct Eq. (11a) is

$$
\lambda \chi_{1} \geq \lambda \chi_{3}+\chi_{2} \chi_{3}+\chi_{1}\left(\frac{4 \eta}{3}-\chi_{4}\right) \geq 0 .
$$

These corrections do not affect the conclusions of the paper. 